\title{
Increased negatively charged nitrogen-vacancy centers in fluorinated diamond
}

\section{Citation}

Cui, Shanying, and Evelyn L. Hu. 2013. Increased Negatively Charged Nitrogen-Vacancy Centers in Fluorinated Diamond. Applied Physics Letters 103(5): 051603.

\section{Published Version}

doi:10.1063/1.4817651

\section{Permanent link}

http://nrs.harvard.edu/urn-3:HUL.InstRepos:12111391

\section{Terms of Use}

This article was downloaded from Harvard University's DASH repository, and is made available under the terms and conditions applicable to Other Posted Material, as set forth at http:// nrs.harvard.edu/urn-3:HUL.InstRepos:dash.current.terms-of-use\#LAA

\section{Share Your Story}

The Harvard community has made this article openly available.

Please share how this access benefits you. Submit a story.

Accessibility 


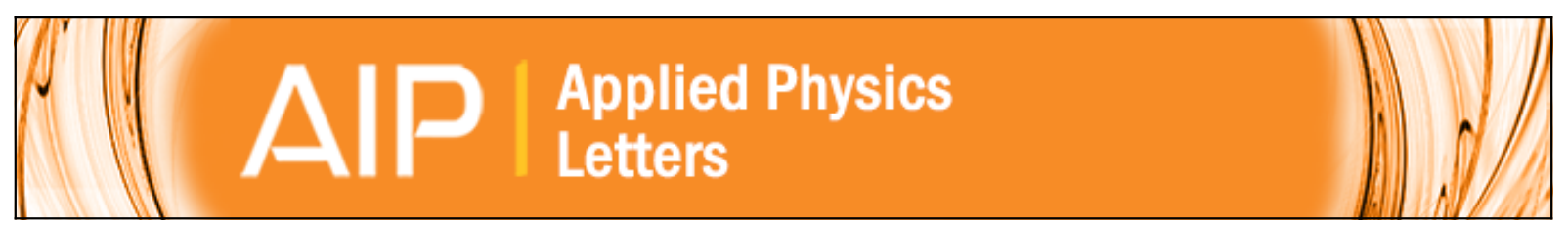

\section{Increased negatively charged nitrogen-vacancy centers in fluorinated diamond}

Shanying Cui and Evelyn L. Hu

Citation: Applied Physics Letters 103, 051603 (2013); doi: 10.1063/1.4817651

View online: http://dx.doi.org/10.1063/1.4817651

View Table of Contents: http://scitation.aip.org/content/aip/journal/apl/103/5?ver=pdfcov

Published by the AIP Publishing

\section{Articles you may be interested in}

Optical manipulation of the charge state of nitrogen-vacancy center in diamond

Appl. Phys. Lett. 103, 013112 (2013); 10.1063/1.4813120

Tunable light emission from nitrogen-vacancy centers in single crystal diamond PIN diodes

Appl. Phys. Lett. 102, 151101 (2013); 10.1063/1.4801871

Conversion of neutral nitrogen-vacancy centers to negatively charged nitrogen-vacancy centers through selective oxidation

Appl. Phys. Lett. 96, 121907 (2010); 10.1063/1.3364135

Influence of a static magnetic field on the photoluminescence of an ensemble of nitrogen-vacancy color centers in a diamond single-crystal

Appl. Phys. Lett. 95, 133101 (2009); 10.1063/1.3238467

Nanopositioning of a diamond nanocrystal containing a single nitrogen-vacancy defect center

Appl. Phys. Lett. 94, 173104 (2009); 10.1063/1.3120558

\section{AlP Re-register for Table of Content Alerts}




\title{
Increased negatively charged nitrogen-vacancy centers in fluorinated diamond
}

\author{
Shanying Cui and Evelyn L. Hu ${ }^{\text {a) }}$ \\ School of Engineering and Applied Sciences, Harvard University, Cambridge, Massachusetts 02138, USA
}

(Received 1 April 2013; accepted 20 July 2013; published online 1 August 2013)

\begin{abstract}
We investigated the effect of fluorine-terminated diamond surface on the charged state of shallow nitrogen vacancy defect centers (NVs). Fluorination is achieved with $\mathrm{CF}_{4}$ plasma, and the surface chemistry is confirmed with x-ray photoemission spectroscopy. Photoluminescence of these ensemble NVs reveals that fluorine-treated surfaces lead to a higher and more stable negatively charged nitrogen vacancy $\left(\mathrm{NV}^{-}\right)$population than oxygen-terminated surfaces. $\mathrm{NV}^{-}$population is estimated by the ratio of negative to neutral charged NV zero-phonon lines. Surface chemistry control of $\mathrm{NV}^{-}$density is an important step towards improving the optical and spin properties of NVs for quantum information processing and magnetic sensing. (C) 2013 AIP Publishing LLC. [http://dx.doi.org/10.1063/1.4817651]
\end{abstract}

The negatively charged nitrogen-vacancy $\left(\mathrm{NV}^{-}\right)$center in diamond is a luminescent point defect with applications in quantum information processing, high sensitivity magnetometry, and biotagging. ${ }^{1}$ The defect can also exist in a neutral charge state $\left(\mathrm{NV}^{0}\right)$, but the attractive optical and spin properties have only been observed in $\mathrm{NV}^{-}$. Recently, significant progress has been made in coupling $\mathrm{NV}^{-}$centers to an external $\operatorname{spin}^{2,3}$ and increasing photon extraction from the diamond lattice. ${ }^{4}$ Both applications require the NVs to be near the surface while maintaining spin coherence and/or optical activity. However, favorable properties, such as $\mathrm{NV}^{-}$luminescence and ESR linewidth, are compromised in shallow NVs. ${ }^{5}$ It is therefore imperative to understand the effect of surface chemistry on the charge state of near-surface NVs and to maximize the number of spin-active $\mathrm{NV}^{-}$states over spin-inactive $\mathrm{NV}^{0}$.

It is well known that hydrogen-termination leads to the depletion of electrons at the surface of the diamond and hence a high $\mathrm{NV}^{0}$ population in shallow NVs. ${ }^{6-9}$ Hydrogen is less electronegative than carbon, and thus a hydrogenterminated diamond surface exhibits a negative electron affinity. Adsorbed water layers on the surface accept electrons, and this electron transfer bends the surface bands upwards, resulting in hole accumulation at the surface and a primarily $\mathrm{NV}^{0}$ population. In contrast, oxygen-terminated surfaces lead to a relatively higher $\mathrm{NV}^{-}$density, which is attributed to the relative electronegativity of oxygen to carbon. The $\mathrm{C}-\mathrm{O}$ surface dipole points in the opposite direction of the $\mathrm{C}-\mathrm{H}$ dipole, resulting in a positive electron affinity for the diamond. Oxygen-termination is currently the state-of-the-art surface termination for diamond. Other chemical terminations to further increase the $\mathrm{NV}^{-}$population have not thoroughly been investigated.

Due to the high electronegativity of fluorine, the C-F bond is more polar than the $\mathrm{C}-\mathrm{O}$ bond. Ab initio calculations of fluorinated diamonds show a stable and full coverage of $\mathrm{F}$, as well as a high electron affinity. ${ }^{10}$ Recently, the fluorinated

\footnotetext{
${ }^{\text {a) }}$ Author to whom correspondence should be addressed. Electronic mail: ehu@seas.harvard.edu
}

diamond electron affinity has been measured experimentally to be $2.56 \mathrm{eV}$, about $0.43 \mathrm{eV}$ higher than for the oxygenterminated surface. ${ }^{11}$ Various methods have been reported in the literature for fluorine-terminating diamond surfaces, including exposure to $\mathrm{XeF}_{2}$ gas, ${ }^{11,12} \mathrm{CF}_{4}$ plasma, ${ }^{13}$ and $\mathrm{CHF}_{3}$ plasma. ${ }^{14}$ However, all prior work on diamond fluorination have been confined to surface chemistry, whereas this work studies the effect of surface fluorination on the charge states of NV centers in bulk diamond.

Experiments were conducted on electronic grade, CVDgrown, (100)-oriented electronic grade diamond (Element6). Surface characterization was carried out with X-ray photoemission spectroscopy (XPS), and charging of the insulating diamond surface was compensated with low energy electrons and an argon ion beam (Thermo Scientific K-alpha). When the stage is not tilted, the XPS signal comes from core electrons within approximately $6 \mathrm{~nm}$ of the surface (three times the mean-free path of electrons in diamond). ${ }^{15}$ Photoluminescence (PL) spectra were collected using $532 \mathrm{~nm}$ laser excitation in a confocal microscope with a $0.5 \mu \mathrm{m}^{2}$ spot size. Prior to implantation, PL was taken at several points on the sample, and no NV luminescence was detected. Diamonds were first implanted with ${ }^{14} \mathrm{~N}$ at $10 \mathrm{keV}$ at $10^{12}$ ions $/ \mathrm{cm}^{2}$ (CORE Systems) and then annealed in vacuum at $800^{\circ} \mathrm{C}$ for $2 \mathrm{~h}$ to create NVs approximately $15 \mathrm{~nm} \pm 5 \mathrm{~nm}$ deep. ${ }^{7}$ To both remove graphitic carbon at the surface and to oxygen-terminate the surface, the samples were cleaned with a boiling solution of 1:1:1 nitric:sulfuric:perchloric acid for $2 \mathrm{~h}$ (boiling triacid). Prior to fluorine-treatment, a photoresist mask (Shipley series) was deposited on half of the sample to protect the oxygentermination (see schematic in Figure 1). Fluorine-treatment was carried out in a Technics RF plasma reactor at $25 \mathrm{sccm}$ $\mathrm{CF}_{4}$ flow rate, $150 \mathrm{~W}$ plasma power, and $300 \mathrm{mTorr}$ chamber pressure for $5 \mathrm{~min}$. In all cases of plasma-treated surfaces, care was taken to avoid etching or otherwise damaging the surface by choosing plasma treatments with no bias voltages and minimizing the interaction time with the plasma. XPS was used to confirm that the process of depositing the photoresist mask and removing the mask with acetone and isopropanol did not affect surface chemistry. 


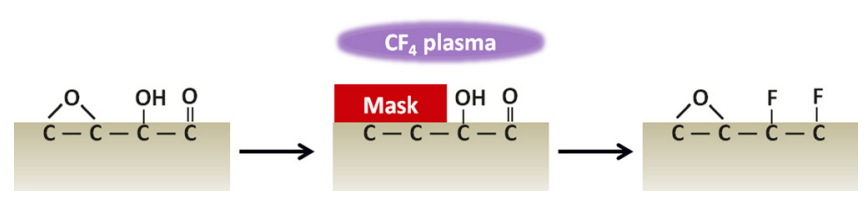

FIG. 1. Schematic of a diamond sample half oxygen-terminated and halffluorine-terminated. Oxygen-termination is achieved with a boiling triacid clean.

The photoelectron spectra of the masked and $\mathrm{CF}_{4}$ plasma-treated half of the same sample are illustrated in Figure 2. The survey spectrum (top inset) reveals $8.5 \%$ oxygen and $91.5 \%$ carbon concentration on the masked side, whereas the side exposed to $\mathrm{CF}_{4}$ shows $7.1 \%$ fluorine, $2.0 \%$ oxygen, and $90.9 \%$ carbon composition. The difference in surface chemistry between the two sides of the sample is further highlighted in the high resolution scan of the $\mathrm{C} 1 \mathrm{~s}$ core electrons and its deconvoluted peaks. Due to the insulating nature of diamond, charging on the surface can shift the

(a)

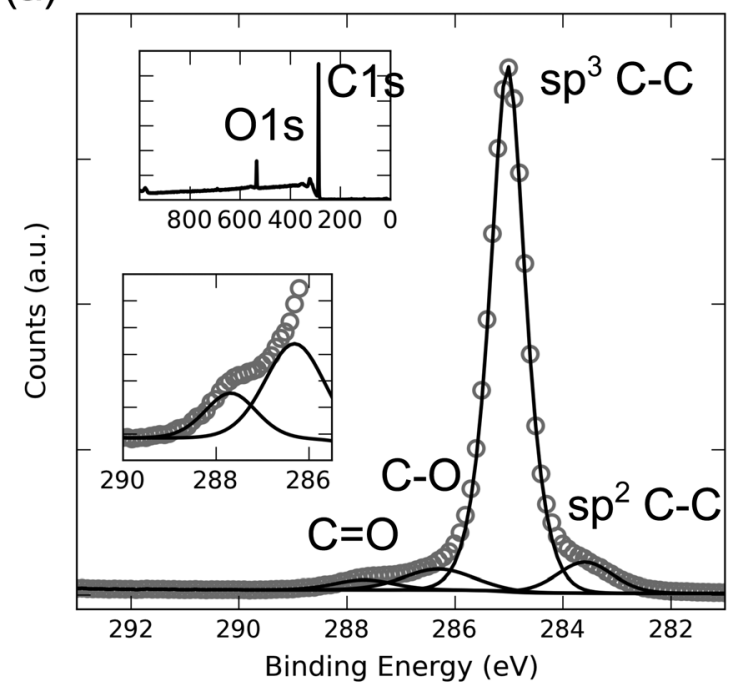

(b)

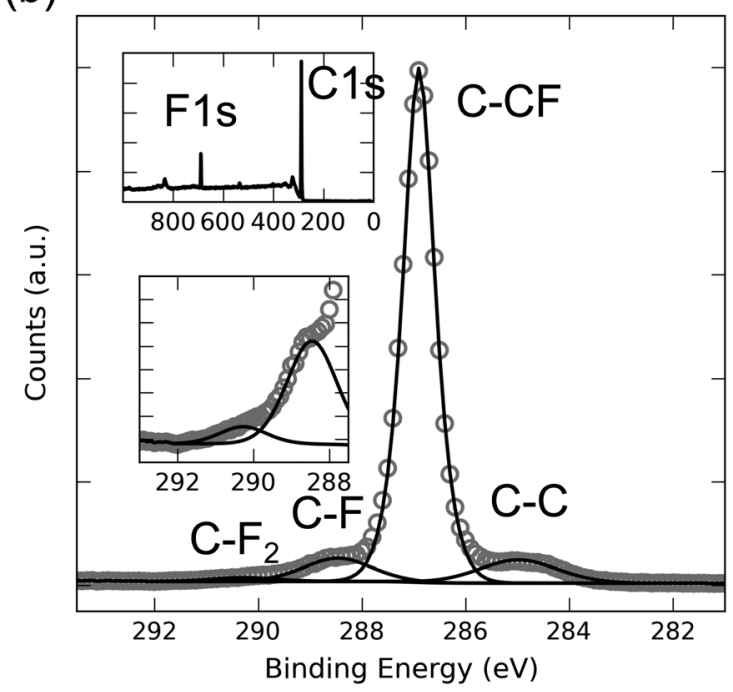

FIG. 2. C 1s core-level photoelectron spectra obtained for (a) oxygen- and (b) fluorine-terminated side of the same sample. Circles correspond to the measured values and solid lines to the fitted peaks. Top inset shows elemental composition of the respective termination. Bottom inset zooms into the higher binding energies to show the peaks more clearly. detected binding energy of electrons, even under a neutralizing electron gun. To mitigate this issue of surface charging induced peak shifts, the spectrum is calibrated by aligning the $\mathrm{sp}^{3} \mathrm{C}-\mathrm{C}$ peak to the known literature value of $285 \mathrm{eV}^{16}$ The calibration is applied to all peaks, such that the relative position of peaks are not affected. The spectrum of the masked side of the sample is calibrated by aligning the main peak to the reported value of $\mathrm{sp}^{3} \mathrm{C}-\mathrm{C}$ bond, shifting the spectrum from the measured peak at $286 \mathrm{eV}$ to $285 \mathrm{eV}$. A graphitic $\mathrm{sp}^{2}$ carbon peak is visible at $283.6 \mathrm{eV}$. Two types of oxidized carbon peaks are visible at 286.3 and $287.7 \mathrm{eV}$, corresponding to $\mathrm{C}-\mathrm{O}$ and $\mathrm{C}=\mathrm{O}$, respectively, evidencing that the masked region is oxygen-terminated. In contrast to the oxygen-terminated side, the main peak on the unmasked side cannot be calibrated to the bulk diamond lattice, since the lowest binding energy peak is $2 \mathrm{eV}$ lower than the main peak, and the $\mathrm{sp}^{2} \mathrm{C}-\mathrm{C}$ bond has only been reported to be between 0.6 and $1.5 \mathrm{eV}$ lower than the $\mathrm{sp}^{3} \mathrm{C}-\mathrm{C}{ }^{14}$ The peak with lowest binding energy has been calibrated from the measured $285.4 \mathrm{eV}$ to the reported $285 \mathrm{eV}$ value. The main peak at $286.9 \mathrm{eV}$ mostly likely reflects a C-CF bond, ${ }^{13,17}$ and the peaks at $288.5 \mathrm{eV}$ and $290.3 \mathrm{eV}$ (better seen in inset) correspond well to $\mathrm{C}-\mathrm{F}$ and $\mathrm{C}-\mathrm{F}_{2}$ literature values. ${ }^{17,18}$ The high concentration of C-CF bonds and the presence of $\mathrm{C}_{-} \mathrm{F}_{2}$ suggest some fluorocarbon deposition from the $\mathrm{CF}_{4}$ plasma, which also has been reported previously in the literature. ${ }^{13}$ We estimate the polymerized fluorocarbon layer to be about $3 \mathrm{~nm}$ thick, given that the C-C bond signal disappears at a $60^{\circ}$ stage tilt, which reduces the analysis depth by half.

The effect of surface termination on NV charge ratio is determined through NV luminescence. A representative PL spectrum in Figure 3(a) shows $\mathrm{NV}^{0}$ and $\mathrm{NV}^{-}$zero phonon lines (ZPL) at 575 and $638 \mathrm{~nm}$, respectively. To estimate the relative probability for the $\mathrm{NV}$ center to be in the $\mathrm{NV}^{-}$state, we analyzed the $\mathrm{NV}^{0}$ and $\mathrm{NV}^{-}$ZPLs individually, by subtracting a linear background, which is attributed to the phonon side bands around the ZPL, and integrating under the peak. Figure 3(b) highlights the ratio of the $\mathrm{NV}^{-} \mathrm{ZPL}$ area to the total NV ZPL area of the same sample over the course of different treatments. Throughout the process, there was no change in overall $\mathrm{NV}$ intensity, only the ratio of $\mathrm{NV}^{-}$and $\mathrm{NV}^{0}$ peaks. While a small fraction of the PL signal can be attributed to intrinsic, deep sub-surface NVs, those intrinsic NVs are equally distributed through the sample, and any differences in the NV ratio due to the deeper NVs should be accounted for in the standard deviation. We started with a boiling triacid-cleaned and oxygen-terminated surface, where PL was taken at multiple points over the whole sample. Error bars here represent standard deviations in the calculated ratio between three to nine spots on the sample. After masking and treating with $\mathrm{CF}_{4}$ plasma, a relative increase of $\mathrm{NV}^{-}$was observed on the fluorine-terminated side. The fluorine-terminated side remained stable after exposure to air for ten days, whereas the $\mathrm{NV}^{-}$ratio on the oxygen-terminated side was further degraded. The lack of stability of the oxygen-terminated surface is not well understood, and further investigation is required. Oxygentermination reflects a mixed chemical state on the surface and can exist as hydroxyl groups, ethers, ketones, or carboxylic acids. Consequently, the oxidation state and the oxygen 
(a)

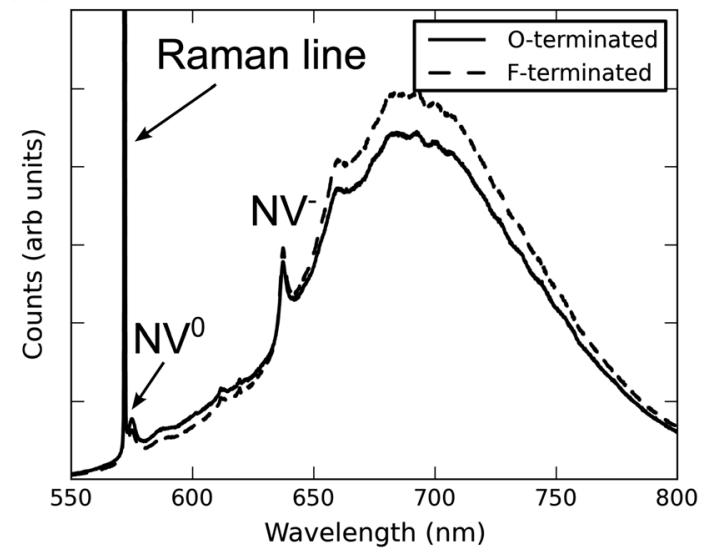

(b)

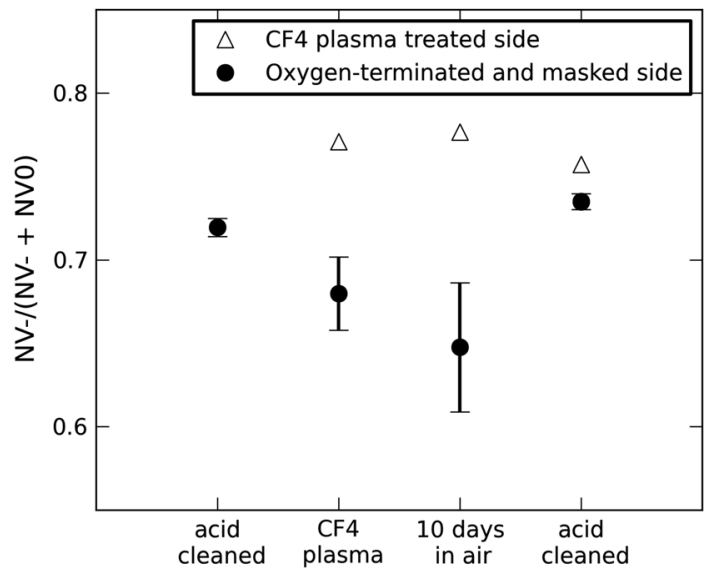

FIG. 3. (a) Representative photoluminescence spectra of the oxygen- and fluorine-terminated side of the sample upon excitation at $532 \mathrm{~nm}$. (b) The ratio of $\mathrm{NV}^{-} \mathrm{ZPL}$ area to the sum of $\mathrm{NV}^{-}$and $\mathrm{NV}^{0} \mathrm{ZPL}$ area of the same sample after 4 subsequent steps: (1) acid cleaned, (2) half protected from (circular markers) and half exposed to (triangular markers) $\mathrm{CF}_{4}$ plasma, (3) left in air for 10 days, and (4) re-oxygenated and cleaned with boiling triacid.

coverage can change upon reaction with moisture in air. Calculations have shown that diamond terminated with hydroxyl groups also exhibits negative electron affinities, ${ }^{19}$ and adsorbed water states are likely to become electron acceptors, as seen with hydrogen-terminated surfaces. The thin polymerized fluorocarbon layer on the $\mathrm{CF}_{4}$ treated side, however, can protect the surface against oxidation and other surface degradations. We restored the whole sample to an oxygen-terminated state by first annealing at $300^{\circ} \mathrm{C}$ to remove the fluorocarbon layer and then treating with boiling triacid. The $\mathrm{NV}^{-} /\left(\mathrm{NV}^{0}+\mathrm{NV}^{-}\right)$ratio on both sides of the sample converged to approximately the initial value.

We compared the effect of hydrogen-, oxygen-, and fluorine-termination without a photoresist mask with a different diamond sample, implanted with ${ }^{14} \mathrm{~N}$ under the same conditions. The sample was first hydrogen-terminated in a $\mathrm{H}_{2}$ plasma by flowing $400 \mathrm{sccm}$ of $\mathrm{H}_{2}$ in a CVD reactor at 60 Torr pressure with a $700 \mathrm{~W}$ plasma power for $3 \mathrm{~min}$. Next, oxygen-termination was achieved with the boiling triacid clean, and finally the sample was fluorine-terminated with $\mathrm{CF}_{4}$ plasma. Between each step, we measured PL at several spots and compared the $\mathrm{NV}^{-}$and $\mathrm{NV}^{0}$ signals. Our experimental results indicate that more electronegative terminations

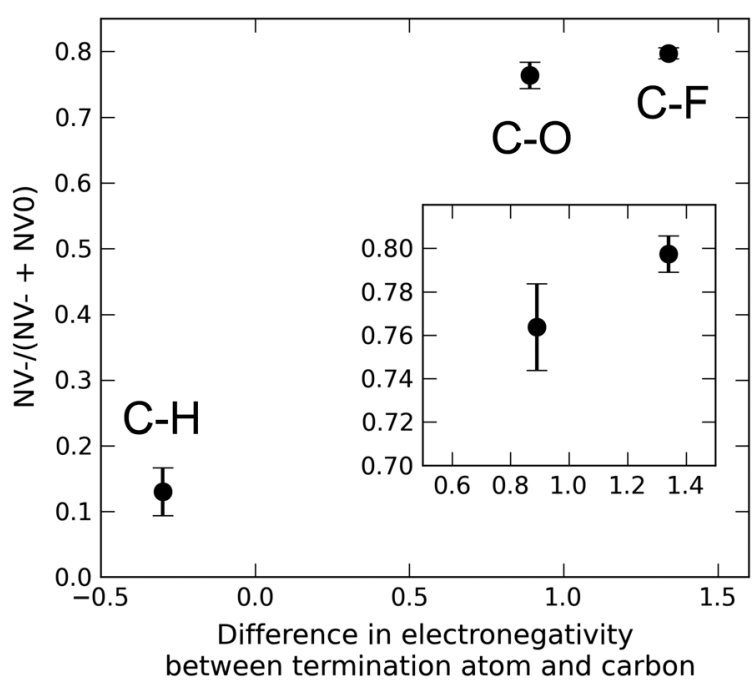

FIG. 4. Ratio of $\mathrm{NV}^{-} \mathrm{ZPL}$ area to the sum of $\mathrm{NV}^{-}$and $\mathrm{NV}^{0} \mathrm{ZPL}$ area for hydrogen-, oxygen-, and fluorine-terminated surfaces. Inset: zoom in for Oand F-termination.

lead to higher relative $\mathrm{NV}^{-}$concentration (Figure 4). Fluorine-termination could induce downward band-bending, ${ }^{11}$ and the strength of the surface dipole can play a significant role in electron density at the surface, thus increasing the relative $\mathrm{NV}^{-}$signal. Additionally, it is important for the surface termination to have good coverage, since dangling bonds can be electron traps. Previous theoretical calculations show that fluorine adsorption energy remains low for all coverages, meaning it is possible to have a full monolayer of $\mathrm{F}$ on a diamond $\langle 100\rangle$ surface, whereas full and stable coverage is less likely for other terminations, such as $\mathrm{Cl}^{10}$

In summary, we demonstrated that fluorine-termination from $\mathrm{CF}_{4}$ plasma treatments yields a higher $\mathrm{NV}^{-}$population than oxygen-termination, which is currently used for all magnetometry and photonic diamond devices. From our photoluminescence data on $\mathrm{H}-, \mathrm{O}-$, and F-terminated surfaces with nearby NVs, we observe a correlation between the surface dipole strength and the ratio of negatively charged NV centers to neutrally charged NVs. Chemical analysis on the surface reveals a polymerized fluorocarbon on the surface that may stabilize and protect the surface, compared to the chemically mixed oxygen-terminated surfaces. Understanding the effect of surface chemistry on NV signal and NV charges is an important step towards building higher sensitivity magnetometers.

This work was supported by the Defense Advanced Research Projects Agency QuASAR program, the Center for Nanoscale Systems (CNS) at Harvard University, and the National Science Foundation Graduate Research Fellowship Program. The authors thank Igor Aharonovich, Jonathan Lee, and Andrew Magyar for stimulating and fruitful discussions.

${ }^{1}$ V. Acosta and P. Hemmer, MRS Bull. 38, 127 (2013).

${ }^{2}$ H. J. Mamin, M. Kim, M. H. Sherwood, C. T. Rettner, K. Ohno, D. D. Awschalom, and D. Rugar, Science 339, 557 (2013).

${ }^{3}$ T. Staudacher, F. Shi, S. Pezzagna, J. Meijer, J. Du, C. A. Meriles, F. Reinhard, and J. Wrachtrup, Science 339, 561 (2013).

${ }^{4}$ T. M. Babinec, B. J. M. Hausmann, M. Khan, Y. Zhang, J. R. Maze, P. R. Hemmer, and M. Loncar, Nat. Nanotechnol. 5, 195 (2010).

${ }^{5}$ B. K. Ofori-Okai, S. Pezzagna, K. Chang, M. Loretz, R. Schirhagl, Y. Tao, B. A. Moores, K. Groot-Berning, J. Meijer, and C. L. Degen, Phys. Rev. B 86, 081406 (2012). 
${ }^{6}$ M. Hauf, B. Grotz, B. Naydenov, M. Dankerl, S. Pezzagna, J. Meijer, F. Jelezko, J. Wrachtrup, M. Stutzmann, F. Reinhard, and J. Garrido, Phys. Rev. B 83, 081304(R) (2011).

${ }^{7}$ K.-M. C. Fu, C. Santori, P. E. Barclay, and R. G. Beausoleil, Appl. Phys. Lett. 96, 121907 (2010).

${ }^{8}$ V. Petráková, A. Taylor, I. Kratochvílová, F. Fendrych, J. Vacík, J. Kučka, J. Štursa, P. Cígler, M. Ledvina, A. Fišerová, P. Kneppo, and M. Nesládek, Adv. Funct. Mater. 22, 812 (2012).

${ }^{9}$ B. Grotz, M. V. Hauf, M. Dankerl, B. Naydenov, S. Pezzagna, J. Meijer, F. Jelezko, J. Wrachtrup, M. Stutzmann, F. Reinhard, and J. A. Garrido, Nat. Commun. 3, 729 (2012).

${ }^{10}$ A. K. Tiwari, J. P. Goss, P. R. Briddon, N. G. Wright, A. B. Horsfall, R. Jones, H. Pinto, and M. J. Rayson, Phys. Rev. B 84, 245305 (2011).

${ }^{11}$ K. J. Rietwyk, S. L. Wong, L. Cao, K. M. O’Donnell, L. Ley, A. T. S. Wee, and C. I. Pakes, Appl. Phys. Lett. 102, 091604 (2013).
${ }^{12}$ J. S. Foord, N. K. Singh, R. B. Jackman, A. Gutierrez-Sosa, S. Proffitt, and K. B. Holt, Surf. Sci. 488, 335 (2001).

${ }^{13}$ A. Denisenko, A. Romanyuk, C. Pietzka, J. Scharpf, and E. Kohn, Diamond Relat. Mater. 19, 423 (2010).

${ }^{14}$ C. Popov, W. Kulisch, S. Bliznakov, G. Ceccone, D. Gilliland, L. Sirghi, and F. Rossi, Diamond Relat. Mater. 17, 1229 (2008).

${ }^{15}$ A. Denisenko, A. Romanyuk, C. Pietzka, J. Scharpf, and E. Kohn, J. Appl. Phys. 108, 074901 (2010).

${ }^{16}$ F. Y. Xie, W. G. Xie, L. Gong, W. H. Zhang, S. H. Chen, Q. Z. Zhang, and J. Chen, Surf. Interface Anal. 42, 1514 (2010).

${ }^{17}$ V. Wheeler, N. Garces, L. Nyakiti, R. Myers-Ward, G. Jernigan, J. Culbertson, C. Eddy, and D. K. Gaskill, Carbon 50, 2307 (2012).

${ }^{18}$ S. Agraharam, D. W. Hess, P. A. Kohl, and S. A. B. Allen, J. Vac. Sci. Technol. A 17, 3265 (1999).

${ }^{19}$ S. Sque, R. Jones, and P. Briddon, Phys. Rev. B 73, 085313 (2006). 